\title{
The development and validation of an augmented video based portable system
}

Ukadike Chris Ugbolue ${ }^{1,3}, *$, Enrica Papi ${ }^{2,3}$,Konstantinos Kaliarntas ${ }^{3}$, Andrew Kerr ${ }^{3}$, Philip J.Rowe ${ }^{3}$

${ }^{1}$ School of Science, Institute for Clinical Exercise \&Health Science, University of the West of Scotland,Hamilton, United Kingdom

${ }^{2}$ Medical Engineering Solutions in OsteoarthritisCentre of Excellence, Imperial College London,London, United Kingdom

${ }^{3}$ Department of Bioengineering, University of Strathclyde, Glasgow, United Kingdom

Introduction and aim:

Three dimensional (3D) motion analysis systems have demonstrated clinical acceptance with the incorporation of active (light emitting diodes) marker and passive (retroreflective) marker measurement systems [1]. Despite the high reliability of 3D motion analysis systems, they remain expensive, complex to operate, time consuming, difficult to understand in terms of data interpretation, and often they are beyond the means and capacity of most rehabilitation services. The need has arisen to develop a 2D video system that meets the requirements of accessibility in terms of cost, operability and portability [2,3]. Within our laboratory a new augmented video based portable system (AVPS) that uses a low cost simple video technique has been developed.

This study reports the concurrent validity of the new AVPS as a potential rehabilitation assessment tool that could be used within the clinical setting.

Patients/materials and methods:

Four stroke patients (three females and one male) aged: $55.3 \pm 9.5$ years, height: $1.67 \pm 0.07 \mathrm{~m}$, and mass: $78.67 \pm 21.2 \mathrm{~kg}$ were recruited. Twelve healthy participants (six females and six males) aged: $28.6 \pm 7$ years, height: $1.71 \pm 0.11 \mathrm{~m}$, and mass: $68.5 \pm 12.1 \mathrm{~kg}$ were recruited. The study was approved by the Department of Biomedical Engineering Ethics Committee and the Departmental Health and Safety Committee. The experimental set up comprised the walkway grid mat, paper bull's eye markers, four photos witches mounted on tripods, a light indicator, video camera, computer with the ProTrainer' system(Sports Motion Inc ${ }^{\circledR}$, Cardiff, CA) software, 3D Vicon Motion Analysis System (Oxford, UK), and a two segment goniometric rig marked with both bull's eye and retroreflective markers. Intra- and inter-rater reliability measurements of the AVPS were performed using three raters. The AVPS output was compared against the 'gold standard' 3D Vicon Motion Analysis System both statically and dynamically using the two segment geometric rig and concurrently validated using the health participants and stroke patients. Kinematic, tibia inclination angles and temporo-spatial gait measurements were obtained. Data were analysed using Intraclass Correlation Coefficient (ICC) for intra-rater reliability to calculate ICC values with 95\% Confidence Intervals. Cronbach's coefficient alpha was used to quantify the inter-rater reliability [4, 5]. Significance level was set to $P=0.05$.

Results:

The AVPS showed good concurrent validity both statically and dynamically when compared against a two segment goniometric rig and the gold standard 3D Vicon Motion Analysis System. Both motion analysis systems showed low levels of intra subject variability. There were no significant differences between the motion analysis systems with respect to the kinematic variables $(P<0.05)$. The results showed a high intra- and inter- rater reliability for both the kinematic and temporo-spatial 
parameters. The lowest ICC value for the kinematic variables was 0.993 . The ICC values ranged from 0.731 to 0.954 for the tibia inclination angles and 0.941 to 0.956 for the temporo-spatial variables.

Discussion and conclusions:

The reliability of the AVPS has been investigated and can be considered as an objective measurement technique that will benefit allied health professionals in their respective clinics and research laboratories. Indeed, the results suggest this 2D video technique of measurement is simple, reliable, effective and easy to implement as a gait outcome measuring system. The system is currently in use as a clinical rehabilitation functional outcome measuring evaluation kit for evaluating stroke patients as part of a clinical trial. Future plans are underway to incorporate the AVPS as an objective measuring tool for evaluating orthopaedic patients as they recover and embark on their rehabilitation training regimes.

\section{Reference}

[1] Davis R. 1988.

[2] Ugbolue et al. 2011.

[3] Ugbolue et al. 2013.

[4] Eliasziw et al. 1994.

[5] Landis JR, Koch GG. 1977. 